\title{
Multidisciplinary Team Approach for Critically Ill Patients
}

\author{
Garg SK ${ }^{1 *}$, Gupta $\mathrm{S}^{2}$ and Sharma $\mathrm{P}^{1}$ \\ ${ }^{1}$ Max Super Speciality Hospital, Saket, New Delhi, India \\ ${ }^{2}$ Dr Baba Saheb Ambedkar Hospital, Rohini, New Delhi, India
}

*Corresponding author: Suneel Kumar Garg, Max Super Speciality Hospital, Saket,

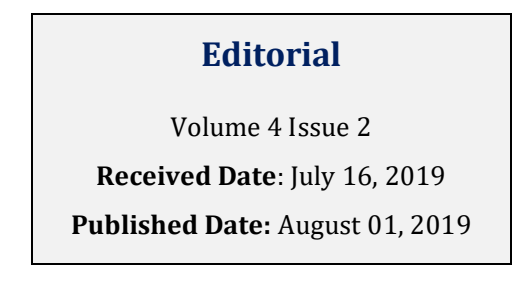

New Delhi, India; Tel: +919873817834; Email: dr_garg@hotmail.com

\section{Editorial}

The ICU serves as a place for monitoring and care of patients with potentially severe physiological instability requiring technical and/or artificial life support. The level of care in an ICU is greater than that available on the floor or intermediate care unit. Number of patients being admitted to Intensive Care Units (ICUs) is increasing each year at various hospitals in the India. These patients suffer from critical illness syndromes such as acute lung injury and sepsis and often are at high risk of mortality ranging from $25 \%$ to $50 \%$.

Approaches to lower ICU mortality include optimizing organisational practices among ICUs, patient-centered culture, strong medical and nursing leadership, effective communication and collaborative approaches for solving problems and managing conflict [1]. For example, a large body of literature indicates that the presence of trained intensivist physicians is associated with improved survival but unfortunately only a minority of ICUs has enough trained intensivists to meet either current or future demand [2].

Hospitals without trained intensivists can still achieve significant reductions in mortality by implementing a multidisciplinary team based approach because patient care depends on the interactions of various providers, especially in the ICU, where critical illness states often result in sudden changes, some of which are lifethreatening. The importance of collaboration and communication and its impact on patient outcomes in the ICU is well recognised by many national and international organisations. The Joint Commission, the American Association of Critical Care Nurses, the Society for Critical
Care Medicine and the National Institutes of Health have also advocated for interdisciplinary communication and collaboration for patient care in the ICU [3-5].

Collaboration is vital in ensuring appropriate care and treatment of the critically ill patient. Multidisciplinary rounds are a major opportunity for ICU team members to share information, perspectives and knowledge, discuss management options, and coordinate patient care. Intensivist-led, inter-professional rounds have been shown to facilitate implementation of best clinical practices such as evidence-based treatments for acute lung injury, sepsis, and prevention of ICU complications.

Pharmacist participation on rounds is associated with fewer adverse drug events and alone may be associated with lower mortality among ICU patients. Communication may facilitate implementation of respiratory therapy and nurse-driven protocols for weaning and sedation, which can reduce duration of mechanical ventilation and shorten ICU length of stay. Recently, Michelle M Kim et al in his multicentric study, investigated the effect of multidisciplinary care team on intensive care unit mortality and concluded that daily rounds by multidisciplinary team are associated with lower mortality among medical ICU patients [6].

\section{Multidisciplinary Team Approach Model}

A number of opportunities have been identified for improving collaboration in the ICU including participation of the multidisciplinary team in grand rounds, research and quality improvement initiatives. 


\section{Anaesthesia \& Critical Care Medicine Journal}

\section{Multidisciplinary Team}

- The Doctor: Every patient will be cared for by the Critical Care team, which is led by an intensivist, a doctor specialized in the care of critically ill patients. These doctors are certified in a medical specialty, such as internal medicine, anesthesiology, surgery or pediatrics, who also receives special education, training, and subspecialty certification, specifically in caring for critically ill patients. Intensivists may be assigned to the ICU on a full-time basis and work with other critical care team members to provide their patients with ongoing and consistent care. All patients will also be cared for by their primary physician. Other physicians may be brought in to consult for specialized advice regarding specific problems. Each physician develops a plan to standardize the care process within the discipline to which he/she belongs. Intensivists coordinate the administrative environment of the ICU by setting policies, developing protocols, and facilitating communication among primary care physicians, specialists, patients, and their families.

- The Nurse: Critical Care nurse spend the majority of time with the patient. Critical care nurses provide a high level of skilled nursing for total patient care and often facilitate communication among all of the people involved in the care of the patient. Their expertise and continuous presence allows early recognition of subtle but significant changes in patient conditions, thereby preventing worsening conditions and minimizing complications that arise from critical illness or injury. Because of their close contact with the family and the patient, critical care nurses often serve as the patient's advocate and become integral to the decision-making process of the patient, family, and critical care team. The nurses and physicians work together to develop a special plan of care for each patient.

- The Clinical Nurse Specialist (CNS): Works with the multidisciplinary team (physicians, nurses, respiratory therapist, social work) to improve patient outcomes by providing expert clinical support to nursing personnel, patients and families.

- The Technician: The technician is similar to the nurse assistant or nurse aide. They have been educated in additional patient care skills, to assist in caring for the Critical Care Patient. Technician will typically work with a nurse in providing different aspects of care. They may draw blood for lab work. They are a vital part of the ICU team.

- Pharmacist or Clinical Pharmacologist: A pharmacist or clinical pharmacologist is a certified specialist in the science and clinical use of medications. The pharmacist with specialty training in the ICU is equipped in recognizing the needs and problems specific to the critical care patient and work with members of the health-care team to foster effective and safe medication therapy.

- Respiratory Therapist: Respiratory therapists work with the critical care team to monitor and promote airway management of the critical care patient. This may include: oxygen therapy, mechanical ventilation (breathing machine) management, aerosol medication therapy, cardio-respiratory monitoring, and patient and caregiver education.

- Physical Therapist or Occupational Therapist - The physical therapist provides services that restore function, improve mobility, relieve pain, and prevent or limit permanent physical disabilities.

- Registered Dietitian: A registered dietician is a vital part of the medical team that consults with physicians, nurses, therapists, and family members in the ICU. The registered dietician works to improve the nutritional health and promotes recovery of the critical care patient.

- Microbiologist: The Microbiologist is also a vital part of team. He helps in catching the various infections and tells sensitivity of drugs for particular microorganism.

- The Social Worker: Another critical care team player is the social worker. These social workers are the experts in helping patients and their families deal with the overwhelming stress associated with critical care. The social worker can help you to better understand and adjust to the patient's condition, as well as relieve any fears or anxiety. They are also available to provide a wealth of information including financial counseling, clergy services, support group referrals, rehabilitation facilities, advanced directives, and ethical concerns.

Our aim should be to have a dedicated team of fulltime intensivists and staff, so the ICU is always covered 24 hours a day, every day. There must be daily formal bedside rounds. Personnel who routinely attend the daily rounds must include the critical care physician and nurses, interdisciplinary specialists, clinical dietician and respiratory therapist. In addition, there should be pharmacy, social work and rehabilitation services for ICU patients. Intensive care is actually incomplete without a dedicated microbiologist who helps us in diagnosis and prescribing antimicrobials. This team coordinates areas of care by establishing guidelines. There must be a monthly meeting to improve communications and to revise clinical 


\section{Anaesthesia \& Critical Care Medicine Journal}

pathways according to feedback from the clinical staff. Goal must be to improve patient and family satisfaction.

\section{Summary}

Multidisciplinary team approach in the ICU is essential for patient focused care, open communication and collaboration are key components in achieving high performance and helps to promote best patient outcomes. Therefore, it is reasonable for hospitals to implement routine multidisciplinary approaches in the ICU when staffing capabilities allow.

\section{References}

1. Zimmerman JE, Shortell SM, Rousseau DM, Duffy J, Gillies RR, et al. (1993) Improving intensive care: a prospective, multicenter study. Crit Care Med 21(10): 1443-1451.

2. Pronovost PJ, Angus DC, Dorman T, Robinson KA, Dremsizov TT, et al. (2002) Physician staffing patterns and clinical outcomes in critically ill patients: a systematic review. JAMA 288(17): 21512162.

3. National Institutes of Health (1983) Consensus conference critical care medicine JAMA 250: 789-804.

4. Joint Commission on Accreditation of Hospitals. Accreditation Manual for Hospitals. 2007; J CAHO Chicago IL.

5. American Association of Critical Care Nurses. Collaborative Practice Model: The Organization of Human Resources in Critical Care Units. AACN: Newport Beach CA.

6. Michelle M Kim, Amber E Barnato, Derek C Angus, Lee F Fleisher, Jeremy M Kahn (2010) The Effect of Multidisciplinary Care Teams on Intensive Care Unit Mortality. Arch Intern Med 170(4): 369-376. 